\title{
The Pattern Efficiency of Energy Transfer Within the Newton's Cradle and Its Practical Usage
}

\author{
David Liu ${ }^{1,}$, , Chenyu Liang ${ }^{2}$ \\ ${ }^{1}$ Civil Engineering Department, University of Toronto, Ontario, Canada \\ ${ }^{2}$ Wuhan British-China School, Wuhan, China \\ Email address: \\ liujz1993@163.com (David Liu),2413932076@qq.com (Chenyu Liang) \\ ${ }^{*}$ Corresponding author
}

\section{To cite this article:}

David Liu, Chenyu Liang. The Pattern Efficiency of Energy Transfer Within the Newton's Cradle and Its Practical Usage. World Journal of Applied Physics. Vol. 6, No. 2, 2021, pp. 24-34. doi: 10.11648/j.wjap.20210602.11

Received: April 15, 2021; Accepted: June 1, 2021; Published: June 4, 2021

\begin{abstract}
In the modern society, the concept of "efficiency" has been emphasized in numerous areas, and is especially critical in discussing the transfer-efficiency of the energy generated. If the concept is not being emphasized in, it is likely that numerous severe consequences would be resulted, for instance, the kinetic energy received by drivers might be massive and even ultimately terminate their lives. The paper targets the factor that affects most in the inertial energy transfer of Newton's Cradle used in the experiment as a subject, getting the information of the actual energy transfer efficiency within the cradle through recording video and locating the position of the balls on a graph paper, analysing the rebounded height level data quantitatively, obtaining the relevant parameters of Cradle such as the mass of the balls and the length of the strings as well as the diameter of the balls through direct measurement. Then, the calculation of the energy loss during the collision would be made and the regression model would be constructed. After that, taking advantage of the hypothesis testing method to testify the significance of the relevant parameter within the Newton's Cradle model. Next, further modifications, which allows a simulation of the energy transfer efficiency of colliding process to be conducted, could be made and the most influencing factor for energy transferring would be revealed and thus find link with the car accident in the method of reducing the damage caused during the incidents.
\end{abstract}

Keywords: Energy Transfer Efficiency, Collision, Newton's Cradle, Linear Regression Data Analysis, Car Accident Scenario

\section{Introduction}

Literature Review

Since the very start of the modern physics was defined, the model of Newton's Cradle has played a significant role in the research about the energy conservation, some researchers had developed relevant scientific methods to find the impact law within the Cradle itself in order to obtain a general mathematical structure of a multi-collision impact law for completely elastic collisions without dissipation, which could ultimately develop into an impact law that conclude most of the circumstances involve inelastic collision [1]. Other researchers still tried to figure out the energy loss pattern during the collisions of the balls and several means of experiment frameworks had been designed and constructed.
Paul Glendinning [2] mentioned in his research that considering the Newton's Cradle in a two-dimensional plane as in $\mathrm{x}$ axis and $\mathrm{y}$ axis can be inadequate, thus the suggestion was to reconstruct the coordinates in a three-dimensional cube as in the $\mathrm{x}$ axis, $\mathrm{y}$ axis and $\mathrm{z}$ axis, which would greatly reduce the error existed in the Z-Axis direction and contribute to improving the accuracy of data collected. Another method implied was to use video software to record and analyse the whole process of the collisions for the balls inside the Newton's Cradle by A. Anissofira et al [3]. Such improvement would allow the researcher to present a clear trajectory frame by frame or as a whole, thus more accurate parameters can be attained. Meanwhile, the structure for the whole experiment would be clearer when the analysis is divided into two views, the macro and the micro views [4]. The macro-perspective would be used to construct the data 
model for the whole experiment and operate quantitative analysis for the data collected while the micro-perspective would mainly focus on the moment that the collisions occurred as the direction and the form of the energy transferred.

A former research about the process of collision illustrated that there are indeed some rules exist as the energy generated by one ball's falling process is not enough to allow two balls at the other side of the cradle to be bounced off, thus the result is clear that the number of balls dropping from one side of the cradle is equal to the number of balls that can be bounced off from the other side of the cradle [5]. Beside the collision itself, relative factors that can determine the energy transfer efficiency was a problem to discuss as well, according to Chenghui WANG [6, 13, 14], the energy transfer efficiency is proportional to the Youngs Modulus as well as the diameter of the ball and inversely proportional to the density of the balls. Another application of the Newton's Cradle model is the mass-spring-damper system in the vehicles for reducing the vibration during the process of driving, this practical usage was inspired by the phenomenon that when the balls at two sides of the cradle move, those are in the middle always remain still $[7,11]$ thus there must be an impact law behind which is even able to be used in the daily life.

\section{Theoretical Guidance and Parameters}

\subsection{Basic Information About the Newton's Cradle Model}

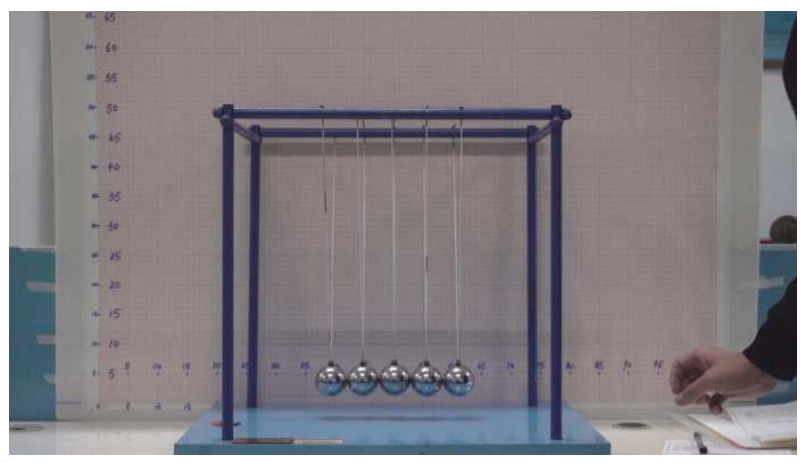

Figure 1. Five ball cradle experiment schematic picture.

The model of the Newton's Cradle is constructed by several identical metal balls (usually five or more) and all are attaching to five equal-length strings (ignorable weight) respectively. In Figure 1, it shows the five-balls Newton's Cradle. When the metal ball at the right end of the device being raised to a certain height and then released, this metal ball will drop and collide with the still ball at its left. Once the ball on one side released from a certain height, it would be given a particular energy to move towards the cradle system. After falling for a period, this ball would collide with the adjacent ball and pass through its energy to the adjacent ball, which would result in the initial ball keeping static in the system. Similarly, all the ball would experience the same phenomenon until the energy transferred to the most peripheric ball and enabled it to repeat the process one more time. The cycle will continuously repeat itself until the inner motion of the Newton's cradle stops. The nature of the question about why model will ultimately stop is there are energy loss due to multiple factors, thus this article will emphasize in determining and analysing the influencing factors about their natures and mechanism about leading to energy loss in the whole moving process.

When focusing the energy loss problem within the Newton's Cradle model, in a macro-perspective, a two-dimensional coordinate system (as x-axis and y-axis) is usually established to locate the position of the balls and make standardized measurement which allows the further calculation of the G.P.E (gravitational potential energy) they possess. As to the micro-perspective, the two most possible factors that can affect the energy transfer efficiency in a grand scale are the friction between the air and the released ball and the energy loss produced by collisions. Base on this, two variables are created in order to indicate the effect of friction and energy loss respectively. One is the number of balls in the cradle and the other is the initial height of the releasing ball. The process of collision itself should be analysed in the mass-spring model as well as the sphere chain model while after the ball swings up. The correlation among the gravitational force, the tension force from the string and the velocity of the ball will be analysed as well. And the further discussion about how the velocity generated by these two forces can influence the energy loss of the system through friction between air and the moving metal ball would be led.

\subsection{The Illustration of the Velocity Possesses by the Moving Balls}

Assume A would be a perfect spherical metal ball on a frictionless plane, of which the mass is $m_{1}$ and velocity is $v_{1}$ moving towards the positive direction. Another perfect spherical metal ball $\mathrm{B}$, possessing a mass of $m_{2}$ and an incident velocity of $v_{2}$, moving towards and collide with $\mathrm{A}$ with both of their barycentre on the same line, as shown in the Figure 2.

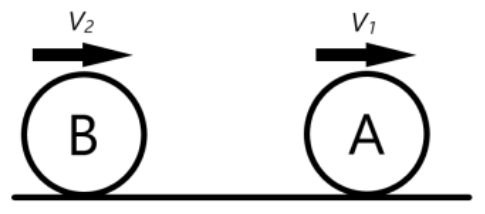

Figure 2. Assumed Model of Collision Balls.

And due to the fact, that during the collision process, the mechanical energy within the system is equilibrium, these following equations which demonstrate the energy conservation and momentum conservation before and after the collision are then able to be suggested in Equation 1 and 2

$$
\frac{1}{2} m v_{1}^{2}+\frac{1}{2} m v_{2}^{2}=\frac{1}{2} m v_{1}^{\prime 2}+\frac{1}{2} m v_{2}^{\prime 2}
$$




$$
m_{1} v_{1}+m_{2} v_{2}=m_{1} v_{1}{ }^{\prime}+m_{2} v_{2}{ }^{\prime}
$$

in which, $v_{1}{ }^{\prime}$ and $v_{2}{ }^{\prime}$ are the velocity of $\mathrm{A}$ and $\mathrm{B}$ after the collision respectively, thus showed in Equation 3 and 4 as below:

$$
\begin{gathered}
v_{1}^{\prime}=\frac{v_{1}\left(m_{1}-m_{2}\right)+2 m_{2} v_{2}}{m_{1}+m_{2}} \\
v_{2}^{\prime}=\frac{v_{2}\left(m_{2}-m_{1}\right)+2 m_{1} v_{1}}{m_{1}+m_{2}}
\end{gathered}
$$

When the masses of two balls are equal, which means $m_{1}=m_{2}$, the conclusion that $v_{1}^{\prime}=v_{2}, v_{1}=v_{2}^{\prime}$. could be drawn. In short, under an ideal circumstance where there is no energy loss exist in any form, the metal ball A will switch its velocity with the metal $\mathrm{B}$. As a result, when the metal ball A is originally still, the metal ball B will then be still after colliding with A. However, the metal ball B would move continuously in the initial velocity of metal ball B before the collision.

The Newton's Cradle model can be considered to be a similar system as the one that illustrated above, which is a sphere chain model made by a series of identical metal balls. Assuming that there is some tiny chink existed between these metal balls as shown in the Figure 3. Thus, the energy transfer process within the cradle is in accord with the quantitative relationship demonstrated above. It indicates that after a series of collisions, the last ball will be bounced off in the same velocity as the released ball at the other end of the cradle while the rest of the metal balls in the system remain still. Under this phenomenon, it can be suggested that the collisions between these metal balls would have sequence as it can be considered as several "one ball to one ball" elastic collisions as the energy contain in the ball before the collision is equal to the energy contained after the collision. Overall, by using this model to interpret the system, the air resistance is the reason why the movement inside the Newton's Cradle would gradually diminish and ultimately terminate.

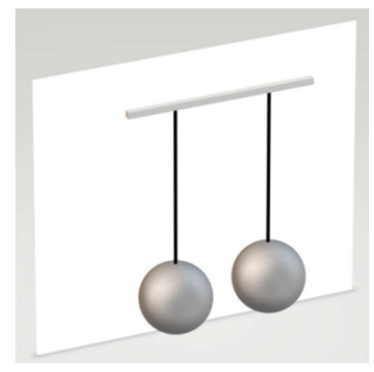

Figure 3. Ideal model of Newton's Cradle.

Nevertheless, in reality, the metal balls within the Newton's Cradle system are closely attached to each other as shown in the Figure 4. It clearly demonstrates the fact that when a metal ball falls and then collide with another metal ball, the collision will influence the energy transfer in the whole system instead of the ball that has engaged in the collision, which directly confront the view of "sequential collisions". As a result, the illustrated ideal sphere chain model cannot be applied to the sphere chain where all balls are closely attached to each other. In another word, the model can only be established when every single ball does not contact with each other initially.

Further illustration is that, as shown in the Figure 5, in a 3-ball Newton's Cradle, when one metal ball is released and collide with the other two closely attached and still metal balls, the ball at the left end of the model does bounce off, yet the ball in middle does not remain completely still, but to fluctuate in a small distance with a low velocity. According to Herrmann et al [8, 12, 15], who first observed the phenomenon that when a metal collided with a sphere chain constructed by a series of balls, the sphere chain itself would break. In detail, he mentioned in his article that if one ball collides with a series of closely attached still balls, the ball which causes the collision will interact with the entire sphere chain, spreading kinetic energy in the sphere chain which would result in the chain to break [9]. Thus, the integrity of the chain will cease to exist and the spreading kinetic energy will be diffused and obtained by each ball in the system by different amount.

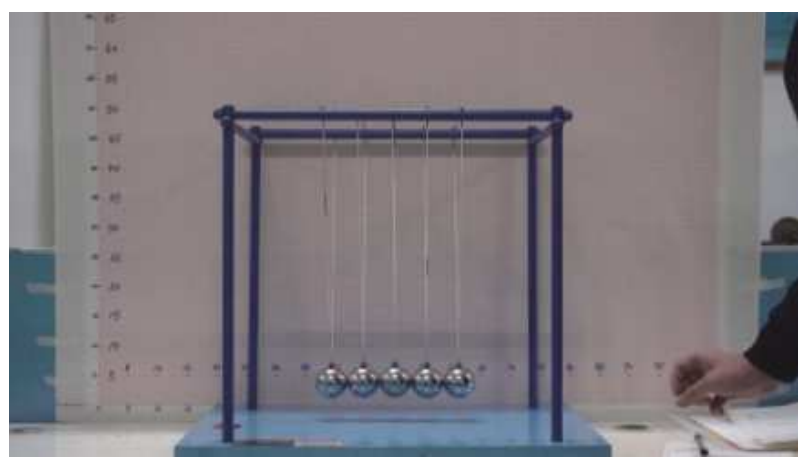

Figure 4. Ball cradle experiment schematic picture.

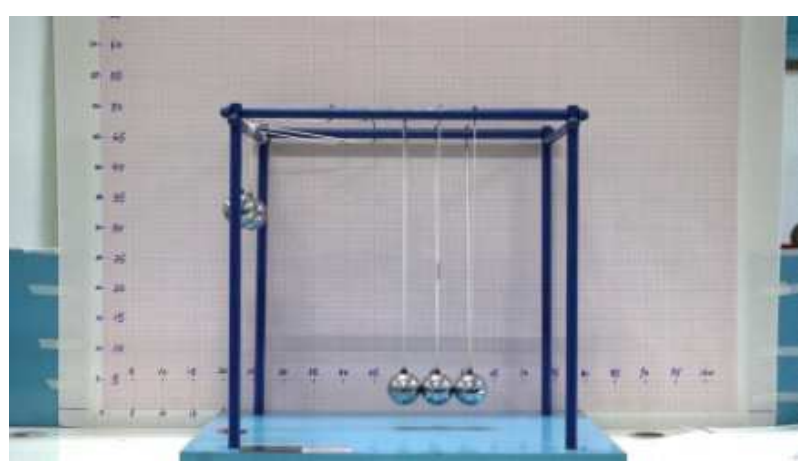

Figure 5. Three ball cradle experiment schematic picture.

\subsection{The Construction of the Mass-spring Model in the System}

The analysis of collisions between balls is usually engaging through the angle of the rigid body dynamics. Due to the fact that the deformation of an object is not considered in analysing process as it cannot be quantified, the analysis toward this model can only be conducted by the conservation theory of both momentum and energy. It would be beneficial for obtaining the general characteristics of the collision 
system, which could leave the phenomenon of energy spreading out in the sphere chain unexplainable.

Nevertheless, in order to analyse the effect of the collision to the sphere chain horizontally, the deformation of the balls during the process of collision cannot be ignored. The analysis can be conducted through the angle of the rigid body dynamics as illustrated below: Simplify the ball as a rigid mass, and use the spring to demonstrate the "collision-partial deformation" relation between balls [10] the rigid mass that are incapable of deforming would store the energy through the motion of the mass while the spring would store its energy though its own deformation. Thus, a model can be constructed as putting a spring in the adjacent balls and deem the balls as mass points and springs which connect to it as shown in the Figure 6 . In this process, the collisions would be considered as the compression of the spring and the relationship between the deformation occurred on balls and the interaction forces among them could be revealed through the mass-spring model illustrated above.

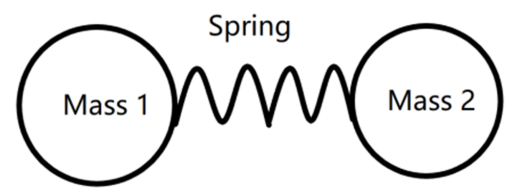

Figure 6. Ideal Mass-spring model.

\section{The Attenuation Mechanism of the Newton's Cradle}

\subsection{The Experiment Framework Construction}

\subsubsection{The Aim of the Experiment}

The purpose of the experiment is to discuss how the various factors affected the decline rate of the vibration within the Newton's Cradle. In order to obtain the relative parameters to conduct analysis, following facilities are required for the experiment: metal balls, thin strings, structure of the Newton's Cradle, $100 \mathrm{~Hz}$ camera for recording, computer for timing, white background plate, graph paper, meter rule, Vernier Calliper, electronic scale for measuring the mass of the balls, stickers, Origin drawing software and etc.

\subsubsection{The Design of the Experiment}

First, the effect of each factor speculated towards the decline in the vibration of the Newton's Cradle would be discussed and the experiments would be operated by controlling two different variables which are the number of the balls within the cradle and initial height of the released ball. Then, the experiment into would be conducted in each group separately due to these different variables and the whole process of the experiment would be recorded by using the $100 \mathrm{~Hz}$ camera. In order to illustrate clearly, the location of the balls a graph paper (A1 paper/index value $1 \mathrm{~mm}$ ) would be used to write coordinates on and to establish a coordinate system which is able to show the initial position of all balls.
The graph paper would be pasted to a white background plate and the final arrangement of the equipment used is shown in Figure below. Establish the Plane coordinate system on the graph paper in which the displacement in vertical direction is represented by the variable of $Y$ and the horizontal displacement is represented by the variable of $X$. Significantly the mass centre of the ball in the middle would be located in a coordinate of $\mathrm{x}=50.0 \mathrm{~cm}$ and $\mathrm{y}=3.5 \mathrm{~cm}$ constantly to avoid possible errors in operation. The research is start with the system of five balls and $3.5 \mathrm{~cm}$ as the initial $\mathrm{y}$ value of the released ball.

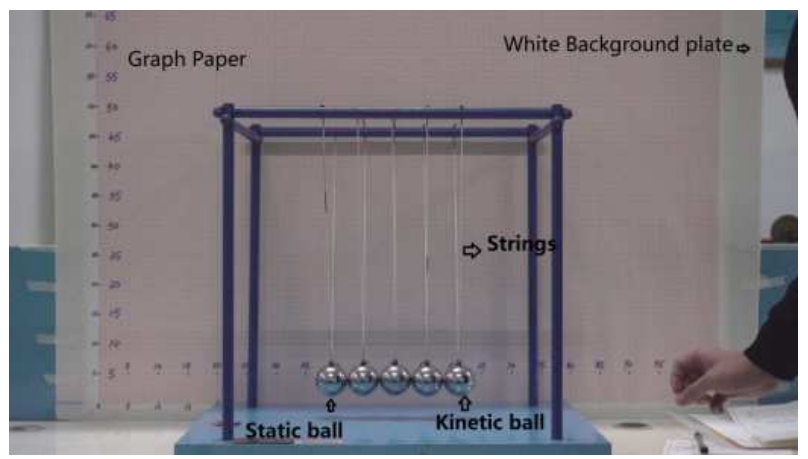

Figure 7. The whole structure of Newton's Cradle.

\subsection{Quantitative Analysis}

\subsubsection{The Influence of the Number of Balls in the Newton's Cradle}

The systems which consist of 2, 3, 4 and 5 diameter balls of which the diameters are $50 \mathrm{~mm}$ would be constructed as shown in Figures 8-11. The metal ball, which is located at the right end of the cradle, would be pulled up as kinetic ball. As showed in the Figures 1-4, it illustrates the change in the height of the bounced-off ball in the system after 1, 2 and 3 collisions in different initial height of the kinetic ball. The centre of the ball in the middle of the cradle has the coordinate of $(50.0 \mathrm{~cm}, 3.5 \mathrm{~cm})$. The height recorded in the graph is the highest point that the mass centre of the ball at both sides of the cradle can reach after the collision. For instance, the mass point of the left end ball would be recorded after the first and the third collision while the mass point of the right end ball would be recoded after the second collision.

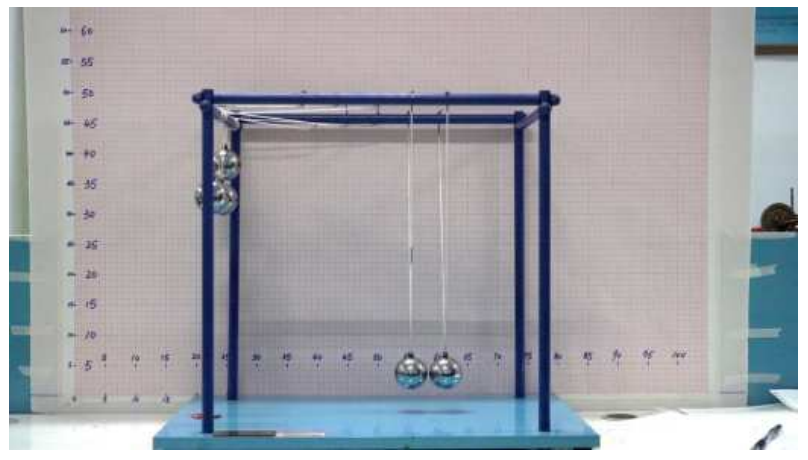

Figure 8. The experiment of Newton's Cradle of 2 balls. 
Table 1. The experiment data of Newton's Cradle of 2 balls.

\begin{tabular}{|c|c|c|c|c|c|}
\hline Number of balls in the cradle & Group Number & Initial height & $1^{\text {st }}$ height & $2^{\text {nd }}$ height & $3^{\text {rd }}$ height \\
\hline \multirow{20}{*}{ 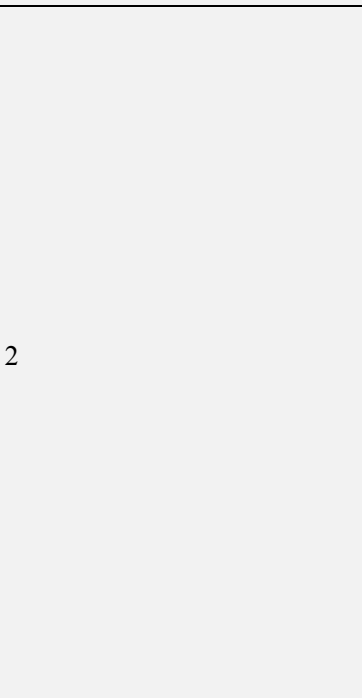 } & \multirow{4}{*}{$2-1$} & 4 & 3.9 & 3.8 & 3.7 \\
\hline & & 3.8 & 3.8 & 3.7 & 3.6 \\
\hline & & 3.8 & 3.8 & 3.7 & 3.6 \\
\hline & & 4 & 4 & 3.9 & 3.8 \\
\hline & \multirow{4}{*}{$2-2$} & 4.5 & 4.4 & 4.3 & 4.2 \\
\hline & & 4.7 & 4.6 & 4.5 & 4.4 \\
\hline & & 4.5 & 4.4 & 4.4 & 4.3 \\
\hline & & 4.6 & 4.5 & 4.4 & 4.3 \\
\hline & \multirow{4}{*}{$2-3$} & 6 & 5.9 & 5.8 & 5.7 \\
\hline & & 6.3 & 6.2 & 6.1 & 6 \\
\hline & & 6.5 & 6.4 & 6.3 & 6.2 \\
\hline & & 6.2 & 6 & 5.9 & 5.9 \\
\hline & \multirow{4}{*}{$2-4$} & 8 & 7.8 & 7.6 & 7.5 \\
\hline & & 8 & 7.9 & 7.8 & 7.7 \\
\hline & & 8 & 7.8 & 7.7 & 7.6 \\
\hline & & 8 & 7.8 & 7.7 & 7.6 \\
\hline & \multirow{4}{*}{$2-5$} & 11 & 10.8 & 10.7 & 10.6 \\
\hline & & 11.5 & 11.3 & 11.1 & 10.9 \\
\hline & & 11.3 & 11.1 & 11 & 10.8 \\
\hline & & 11.4 & 11.2 & 11 & 10.8 \\
\hline
\end{tabular}

Table 2. The experiment data of Newton's Cradle of 3 balls.

\begin{tabular}{|c|c|c|c|c|c|}
\hline Number of balls in the cradle & Group Number & Initial & 1 st & 2nd & 3rd \\
\hline \multirow{17}{*}{3} & \multirow{4}{*}{$3-1$} & 4 & 3.9 & 3.8 & 3.7 \\
\hline & & 3.8 & 3.8 & 3.7 & 3.7 \\
\hline & & 3.8 & 3.7 & 3.7 & 3.6 \\
\hline & & 3.8 & 3.8 & 3.7 & 3.6 \\
\hline & \multirow{3}{*}{$3-2$} & 4.5 & 4.4 & 4.4 & 4.3 \\
\hline & & 4.5 & 4.4 & 4.3 & 4.2 \\
\hline & & 4.7 & 4.6 & 4.5 & 4.4 \\
\hline & \multirow{4}{*}{$3-3$} & 6 & 5.9 & 5.8 & 5.7 \\
\hline & & 6.2 & 6.1 & 6 & 5.9 \\
\hline & & 6.6 & 6.4 & 6.3 & 6.2 \\
\hline & & 6.1 & 6 & 5.9 & 5.8 \\
\hline & \multirow{3}{*}{$3-4$} & 8.1 & 7.9 & 7.7 & 7.5 \\
\hline & & 8 & 7.8 & 7.7 & 7.5 \\
\hline & & 8.3 & 8.1 & 7.9 & 7.7 \\
\hline & \multirow{3}{*}{$3-5$} & 11.2 & 10.9 & 10.7 & 10.5 \\
\hline & & 11.5 & 11.1 & 10.8 & 10.6 \\
\hline & & 11.5 & 11.2 & 10.9 & 10.7 \\
\hline
\end{tabular}

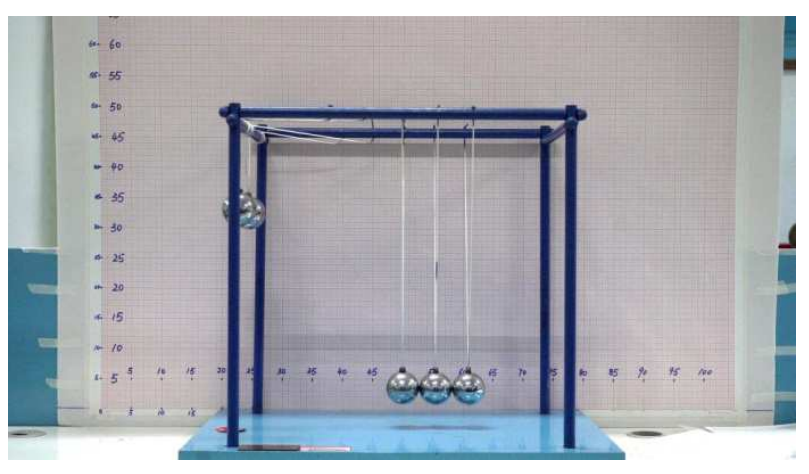

Figure 9. 3 balls experiment of Newton's Cradle.

The majority of data collected reveals that the conclusion made through the experiment data is coincide with the prediction suggested before the experiment. When the number of the metal balls is small, the kinetic energy distributed among the balls in the middle would be relatively less, which result in a slow declination in the vibration rate within the Newton's Cradle. On the contrary, when the number of balls in the cradle is large, the kinetic energy distributed among the balls in the middle of the cradle would be relatively large which result in a fast declination in the vibration rate within the Newton's Cradle.

\subsubsection{The Influence of the Initial Height of the Kinetic Ball}

Use the single balls cradle as the sample (In order to reduce error from calculating the energy loss during the collision between balls) and lift up the metal ball at the right end of the system at the height of $3.8 \mathrm{~cm}, 4.5 \mathrm{~cm}, 6.0 \mathrm{~cm}$, $8.0 \mathrm{~cm}$ and $11.5 \mathrm{~cm}$ to conduct the experiment respectively. The initial state before releasing the kinetic ball is shown in Figure 12. The experiment varies the velocity of the released metal ball just before the collision by changing the initial height of it, thus observe the effect on the declination of 
vibration within the Cradle. The data collected from the experiment is shown in the Figure 5.

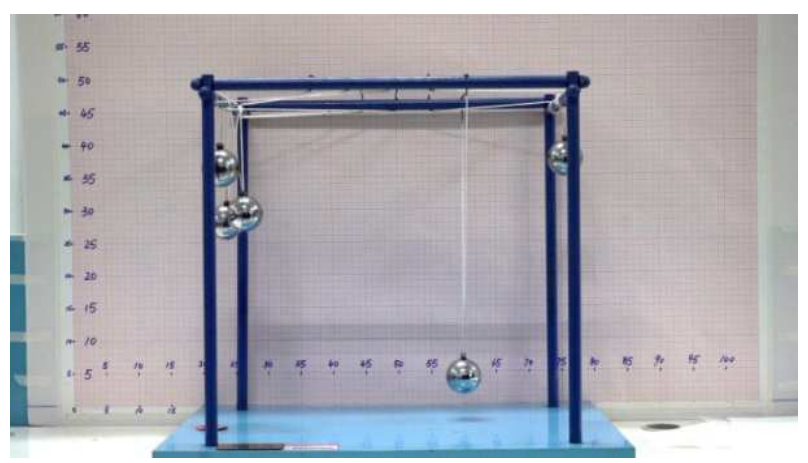

Figure 10. Single ball pendulum experiment.
It is obviously shown from the experiment that as the initial height of the kinetic ball increases, the rate of declination in the vibration within the Newton's Cradle increases as well. Such phenomenon could be interpreted as following, after the initial height of the kinetic ball increases, the Gravitational Potential Energy of it increases as well which leads to a further increase in the Kinetic Energy possessed by the ball when reaching the mid-point of the Cradle. So, ceteris paribus in other qualities of the ball, the velocity of the ball at the mid-point would increase and result in more energy loss due to air friction as the latter is proportional to the velocity of the ball. Thus, the rate of declination within the Cradle would decrease.

Table 3. The experiment data of Newton's Cradle of 2 balls.

\begin{tabular}{|c|c|c|c|c|c|}
\hline Number of balls in the cradle & Group Number & Initial & 1st & 2nd & 3rd \\
\hline \multirow{20}{*}{1} & \multirow{4}{*}{$1-1$} & 3.8 & 3.8 & 3.8 & 3.7 \\
\hline & & 3.8 & 3.8 & 3.8 & 3.7 \\
\hline & & 4 & 4 & 4 & 3.9 \\
\hline & & 3.9 & 3.9 & 3.9 & 3.7 \\
\hline & \multirow{4}{*}{$1-2$} & 4.5 & 4.4 & 4.3 & 4.3 \\
\hline & & 4.5 & 4.4 & 4.4 & 4.3 \\
\hline & & 4.5 & 4.4 & 4.4 & 4.4 \\
\hline & & 4.5 & 4.4 & 4.3 & 4.3 \\
\hline & \multirow{4}{*}{$1-3$} & 6 & 5.9 & 5.9 & 5.8 \\
\hline & & 6.5 & 6.4 & 6.3 & 6.2 \\
\hline & & 6 & 5.9 & 5.9 & 5.8 \\
\hline & & 6 & 5.9 & 5.8 & 5.7 \\
\hline & \multirow{4}{*}{$1-4$} & 8 & 7.9 & 7.8 & 7.7 \\
\hline & & 8 & 7.9 & 7.7 & 7.6 \\
\hline & & 8 & 7.8 & 7.7 & 7.6 \\
\hline & & 8 & 7.9 & 7.8 & 7.7 \\
\hline & \multirow{4}{*}{$1-5$} & 11.5 & 11.3 & 11.1 & 11 \\
\hline & & 11.5 & 11.3 & 11.2 & 11 \\
\hline & & 11 & 10.8 & 10.7 & 10.6 \\
\hline & & 11.5 & 11.3 & 11.1 & 10.9 \\
\hline
\end{tabular}

\section{Further Data Analysis}

In order to obtain a more convincing result, further data quantitative analysis has to be made. Thus, the following 10 graphs would illustrate the trend of energy loss when the initial height or the number of the balls within the Cradle changes. To be more specific, among these 10 graphs, the first five graphs are made by considering the number of the balls in the Cradle as the variable while the last five are made by choosing the initial height of the released ball as a variable. All graphs are drawn due to a total of 300 groups of observed experimental data obtained as for each position that was needed to be measured, the height of the balls was measured for four times and then averaged in order to obtain a final value.

\subsection{Study on Initial Ball Height and Energy Conversion Efficiency}

With 2 balls, swing for 3 times at $4.5 \mathrm{~cm}$ initial heights, as shown in the figure below

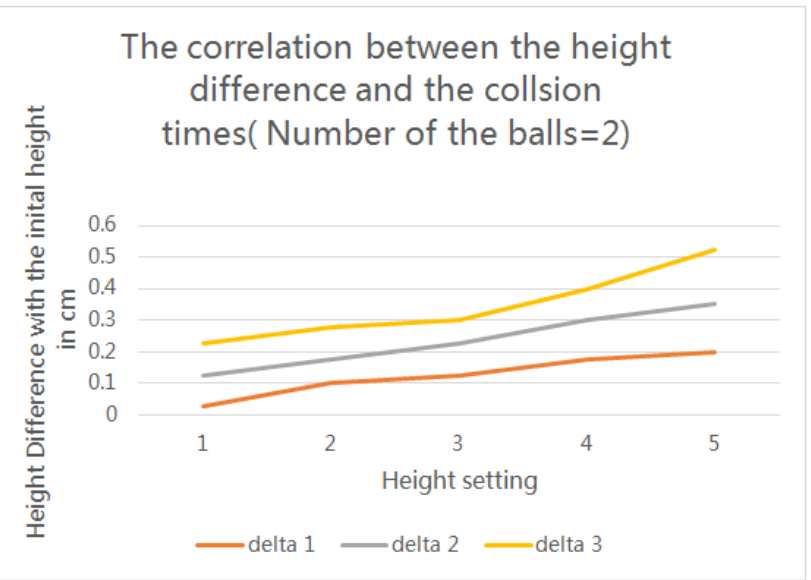

Figure 11. The correlation between the height difference and the collision times (Numbers of the ball=2).

As can be seen from the figure, there are only two small balls, one of which will collide when it swings. As the initial height increases at any time, the height of the first impact will 
gradually decrease. There were 1 impact, 2 impact and 3 impact, all of which showed the same trend. It shows that when the number of balls remains the same, the initial height increases at any time, and the height of the swing after impact decreases more and more, indicating that the energy conversion efficiency decreases.

Similarly, the curves for the system of 1 ball, 3 balls, 4 balls and 5 balls also showed a similar trend to the two-ball cradle experiment as well. Having a specific comparison about these curve and figure, the following conclusion could be obtained: First, the curve for the system of 3 balls to 5 balls presented similar trends and shapes. However, the curve for the system of 2 balls is different, especially for the line of delta 3. Second, each line in the figure would contains at least a turning point. After this point, the rate of increasing for the line would be greatly improved. Comparing the turning point in each line, curve and graph, the conclusion that the increasing for the number of balls in the system would lead to the early appearing of the turning points in the line.

\subsection{Study on the Trend of Energy Conversion and Number of Balls}

When the initial height is $3.8 \mathrm{~cm}$ and different number of balls swing for 3 times, see the following figure:

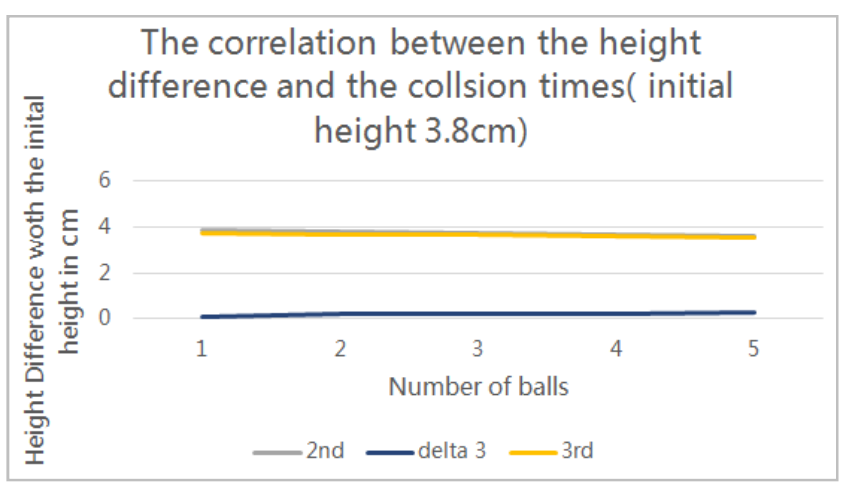

Figure 12. The correlation between the height difference and the collision times (Initial height $=3.8 \mathrm{~cm}$ ).

As can be seen from the figure, when the initial height is 3.8 $\mathrm{cm}$, collision will occur when a ball swing. With the increase of the number of balls at any time, the drop in the bouncing height of the ball passed to the other side gradually increases after the impact. There was the $1^{\text {st }}$ impact, the $2^{\text {nd }}$ impact and the $3^{\text {rd }}$ impact irrespectively. All of them showed the almost same trend. It shows that when the initial height of the balls remains unchanged, the height of the swing after impact decreases more and more with the increase of the number of balls, which indicates that the energy conversion efficiency decreases.

Similarly, the curve for the initial height of kinetic ball for $4.5 \mathrm{~cm}, 6 \mathrm{~cm}, 8 \mathrm{~cm}$ and $11 \mathrm{~cm}$ also showed similar trend of energy loss pattern as well. Through the comparison of these graphs, the conclusions below could be revealed. First, the initial height for the kinetic ball would have great impact on the shape of the curve. However, once the initial height increases to the value of $8 \mathrm{~cm}$, the shape would almost keep constant and no vital changes would occur in this condition. Moreover, when the initial height of the balls remains unchanged, the height of the swing after impact decreases more and more with the increase of the number of balls, which indicates that the energy conversion efficiency decreases.

\subsection{Hypothesis Testing Preparation}

Based on the guidance for the application of hypothesis testing, it is compulsory to make the following two of Proposed hypotheses:

1. When the number of pellets remains constant, the initial height of pellets is negatively correlated with the energy conversion efficiency;

2. When the initial height is constant, the number of balls in the experiment is negatively correlated with the energy conversion efficiency

And before conducting the quantitative analysis, enter the variable Time, which represents the number for the time of collision, occurred within the Cradle.

\subsection{Further Quantitative Analysis of Initial Ball Height and Energy Conversion Efficiency}

1. For the system of one ball, which swings three times, the correlation analysis has been conducted and relative parameters could be obtained and listed below in Graph.

Table 4. The parameters for the quantitative analysis for the Newton's cradle (Initial Ball Height \& Energy Conversion Efficiency).

\begin{tabular}{|c|c|c|c|c|}
\hline \multicolumn{5}{|c|}{ Correlations } \\
\hline & & Initial & Time & Delta \\
\hline \multirow{3}{*}{ Initial } & Pearson Correlation & 1 & .000 & $.748^{* *}$ \\
\hline & Sig. (2-tailed) & & 1.000 & .001 \\
\hline & $\mathrm{N}$ & 15 & 15 & 15 \\
\hline \multirow{3}{*}{ Time } & Pearson Correlation & .000 & 1 & $.550^{*}$ \\
\hline & Sig. (2-tailed) & 1.000 & & .034 \\
\hline & $\mathrm{N}$ & 15 & 15 & 15 \\
\hline \multirow[t]{2}{*}{ Delta } & Sig. (2-tailed) & .001 & .034 & \\
\hline & $\mathrm{N}$ & 15 & 15 & 15 \\
\hline
\end{tabular}

**. Correlation is significant at the 0.01 level (2-tailed).

*. Correlation is significant at the 0.05 level (2-tailed). 
Correlation coefficient between Initial and delta was 0.748 , SIG 0.001 was less than 0.05 , which passed the significance test. The Initial height is strongly negatively correlated with the energy transmission efficiency, and the delta represents the attenuation of energy. As for the regression analysis, it presented in the following contents.

Table 5. The parameters for the regression analysis of the Newton's cradle (Initial Ball Height \& Energy Conversion Efficiency).

\begin{tabular}{lllll}
\hline Model Summary & & & & \\
\hline Model & R & R Square & Adjusted R Square & Std. Error of the Estimate \\
\hline 1 & $.928^{\mathrm{a}}$ & .862 & .839 & .053958 \\
\hline
\end{tabular}

a. Predictors: (Constant), time, Initial

\begin{tabular}{llllll}
\hline ANOVA $^{\mathbf{a}}$ & & & & & \\
\hline Model & & Sum of Squares & df & Mean Square & F \\
\hline \multirow{3}{*}{1} & Regression & .218 & 2 & .109 & 37.506 \\
& Residual & .035 & 12 & .003 & $.000^{\mathrm{b}}$ \\
& Total & .253 & 14 & & \\
\hline
\end{tabular}

a. Dependent Variable: delta

b. Predictors: (Constant), time, Initial

\begin{tabular}{|c|c|c|c|c|c|c|}
\hline \multicolumn{7}{|c|}{ Coefficients $^{\mathrm{a}}$} \\
\hline \multirow{2}{*}{ Model } & & \multicolumn{2}{|c|}{ Unstandardized Coefficients } & \multirow{2}{*}{$\begin{array}{l}\text { Standardized Coefficients } \\
\text { Beta }\end{array}$} & \multirow{2}{*}{$\mathbf{T}$} & \multirow{2}{*}{ Sig. } \\
\hline & & $\mathbf{B}$ & Std. Error & & & \\
\hline \multirow{3}{*}{1} & (Constant) & -.227 & .051 & & -4.467 & .001 \\
\hline & Initial & .036 & .005 & .748 & 6.980 & .000 \\
\hline & time & .088 & .017 & .550 & 5.128 & .000 \\
\hline
\end{tabular}

a. Dependent Variable: delta

The goodness of fit $\mathrm{R}$ squared of the model is 0.928 , indicating that the model has a good fit. SIG in ANOVA is less than 0.05 , indicating that the model itself has passed the significance test; In parameter estimation, the Sig values of constant term, Initial and delta were all less than 0.05 , indicating that the parameter estimation passed the significance test. And the equation for the regression line could be obtained for one ball in the system and swing for 3 times is

Delta $=0.88 *$ time $+0.036 *$ Initial -0.227

Similarly, the regression line for the system, which contains 3,4 and 5 balls could also be obtained as showed above, which are 2 to 5 balls respectively:

Delta $=0.11 *$ time $+0.03 *$ Initial -0.19

Delta $=0.13 *$ time $+0.057 *$ Initial -0.363
Delta $=0.143 *$ time $+0.072 *$ Initial -0.447

Delta $=0.182 *$ time $+0.084 *$ Initial -0.497

So, having a specific analysis about the variables in the experiments and the regression mode, it is interesting to note that the weight of time would be greatly improved for the value of delta in the system, when the number of balls in the system increases. Moreover, on the contrary, the weight of initial height for the value of delta in the regression equation would be different.

\subsection{Further Quantitative study on the Number of ball and Energy Conversion Efficiency in the Experiment}

For the group of initial height of 3.8, 3 times of rocking. Correlation analysis

Table 6. The parameters for the quantitative analysis of the Newton's cradle (Numbers of balls \& Energy Conversion Efficiency).

\begin{tabular}{|c|c|c|c|c|}
\hline \multicolumn{5}{|l|}{ Correlations } \\
\hline & & Number_ball & time & delta \\
\hline \multirow{3}{*}{ Number_ball } & Pearson Correlation & 1 & .000 & $.663^{* *}$ \\
\hline & Sig. (2-tailed) & & 1.000 & .007 \\
\hline & $\mathrm{N}$ & 15 & 15 & 15 \\
\hline \multirow{3}{*}{ time } & Pearson Correlation & .000 & 1 & $.689^{* *}$ \\
\hline & Sig. (2-tailed) & 1.000 & & .005 \\
\hline & $\mathrm{N}$ & 15 & 15 & 15 \\
\hline \multirow[t]{2}{*}{ delta } & Sig. (2-tailed) & .007 & .005 & \\
\hline & $\mathrm{N}$ & 15 & 15 & 15 \\
\hline
\end{tabular}

**. Correlation is significant at the 0.01 level (2-tailed).

Correlation coefficient between Initial and delta was 0.663 , SIG 0.001 was less than 0.05 , which passed the significance test. The Initial height showed a strong positive correlation with delta, and delta represented energy attenuation, indicating that the Initial height had a strong negative correlation with energy transmission efficiency. 
Table 7. The parameters for the regression analysis of the Newton's cradle (Numbers of balls \& Energy Conversion Efficiency).

\begin{tabular}{lllll}
\hline Model Summary & & & \\
\hline Model & R & R Square & Adjusted R Square & Std. Error of the Estimate \\
\hline 1 & $.956^{\mathrm{a}}$ & .914 & .899 & .029226 \\
\hline
\end{tabular}

a. Predictors: (Constant), time, Number_ball

\begin{tabular}{lllllll}
\hline ANOVA $^{\mathbf{a}}$ & & & & & \\
\hline Model & & Sum of Squares & df & Mean Square & F & Sig. \\
\hline \multirow{3}{*}{1} & Regression & .108 & 2 & .054 & 63.415 & $.000^{\mathrm{b}}$ \\
& Residual & .010 & 12 & .001 & & \\
& Total & .119 & 14 & & & \\
\hline
\end{tabular}

a. Dependent Variable: delta

b. Predictors: (Constant), time, Number_ball

\begin{tabular}{|c|c|c|c|c|c|c|}
\hline \multicolumn{7}{|c|}{ Coefficients $^{\mathrm{a}}$} \\
\hline \multirow{2}{*}{ Model } & & \multicolumn{2}{|c|}{ Unstandardized Coefficients } & \multirow{2}{*}{$\begin{array}{l}\text { Standardized Coefficients } \\
\text { Beta }\end{array}$} & \multirow{2}{*}{$\mathbf{t}$} & \multirow{2}{*}{ Sig. } \\
\hline & & B & Std. Error & & & \\
\hline \multirow{3}{*}{1} & (Constant) & -.138 & .026 & & -5.406 & .000 \\
\hline & Number_ball & .042 & .005 & .663 & 7.809 & .000 \\
\hline & time & .075 & .009 & .689 & 8.115 & .000 \\
\hline
\end{tabular}

a. Dependent Variable: delta

The goodness of fit $\mathrm{R}$ squared of the model is 0.914 , indicating that the model is well fitted. SIG in ANOVA is less than 0.05 , indicating that the model itself has passed the significance test. In parameter estimation, the Sig values of constant term, Initial and delta were all less than 0.05 , indicating that the parameter estimation passed the significance test. So, based on the analysis and calculation above, the regression equation is:

\section{Delta $=0.075 *$ time $+0.042 *$ Initial -0.138}

Similarly, the regression equation for the initial height 3.8 , $4.5,6.0,8.0$ and $11.0 \mathrm{~cm}$ of the kinetic ball could be obtained and showed as following respectively:

Delta $=0.08 *$ time $+0.01 *$ Number of ball -0.007

Delta $=0.11 *$ time $+0.046 *$ Number of ball -0.112

Delta $=0.167 *$ time $+0.067 *$ Number of ball -0.18

Delta $=0.22 *$ time $+0.131 *$ Number of ball -0.271

So, having a specific analysis about the variables in the experiments and the regression mode, it is interesting to note that the weight of time would be greatly improved for the value of delta in the system, when the number of balls in the system increases. Moreover, similarly, the weight of number of balls in the system would determine the value of delta in the regression equation would be different, with the increasing of initial height of the kinetic ball in the experiment.

\section{Conclusion}

In general, the first part of my quantitative analysis which only involve the usage of graphs showed a equivocate trend of the energy loss which is that the more ball a cradle possesses or higher the kinetic ball is, the more energy loss during the moving process. And through further cross-reference of all the graphs, it was to say that the trend of energy loss can only be clear when there is more than or equal to two balls in the system which can be an explanation of why the Newton's Cradle used for academic research is usually with five or even more balls. A further assumption of this can be that when there are more impacts occur as more cars involved in a car accident, the energy loss trend may be more obvious to be used and conduct further experiment. When it came to the further data analysis part, a new factor time was introduced into the regression modelling construct and the result was illustrated through several equation that can show the quantitative relationship between each variable. And it was to say that the time is the factor that can determine the energy loss the most, as an introduction to the reality, the it can be interpreted in reality that as the shorter time taken for car to impact which can be combined with another factor-Initial height of the kinetic ball that the higher velocity the moving car/ball possesses just before the collision (Illustrated in the former concluded quantitative relationship), the lower energy loss during the process and thus the greater energy impact received by the driver and cause larger damage to human body. Apart from the factor of initial height the number of balls in the cradle can also be interpreted as the number of cars involved or the number of collisions happened in the car crash. So as illustrated in the equation of regression modelling construct, an increase in the number of collisions between cars/balls can effectively reduce the energy transfer efficiency during the collision process which allow fewer energy transferred to drivers in each car and cause less damage to their body. Thus, the overall suggestion from the conclusion is that producers may can consider about longer the total structure especially the front part or the backward part of the vehicles to or using better materials or other methods to improve the safety air bag to increase the time of the collision process and thus decrease the energy received by drivers. Apart from what can be done by the producers, the government may enable speed restriction on busy roads which can forbidden cars to go too fast so even if there are cars 
collides with each other, their velocity can be low and more energy loss during the transfer and less received by drivers.

\section{Appendix}

1. Former researches

Apart from the experiment used methods, there were researches about the collision pattern within the two-balls Newton's Cradle as well. According to Rod Cross et al [10], the collisions inside the two-ball Newton's Cradle were nearly elastic as there were little energy loss during the collisions, however, it was suggested that the phenomenon of two balls "wobble" which means the two single ball pendulums combined into a two balls pendulum exist in almost every single experiment, thus it was said that the energy loss during such phenomenon can be hard to measure and the phenomenon itself can exist in any Newton's Cradle as long as the number of the balls exceed two. Still, many researchers chose to combine the model of single pendulum into the research of the Newton's Cradle [11] as they were actually convinced that the Newton's Cradle is a formation of several single non-linear pendulum model which allow the researchers to cite the equations concluded through analysing the single pendulum and use them to make ideal calculation in order to compare with the data collected in the experiment. In addition, few have ever focus on the energy loss during the collision of the five or four balls as most believed the error of the data collected can be massive [12] so the trend concluded through the data can deviate from what it should be and even wrong.

2. Other sets of data collected from the experiment:

Table 8. The experiment data of Newton's Cradle of 4 balls.

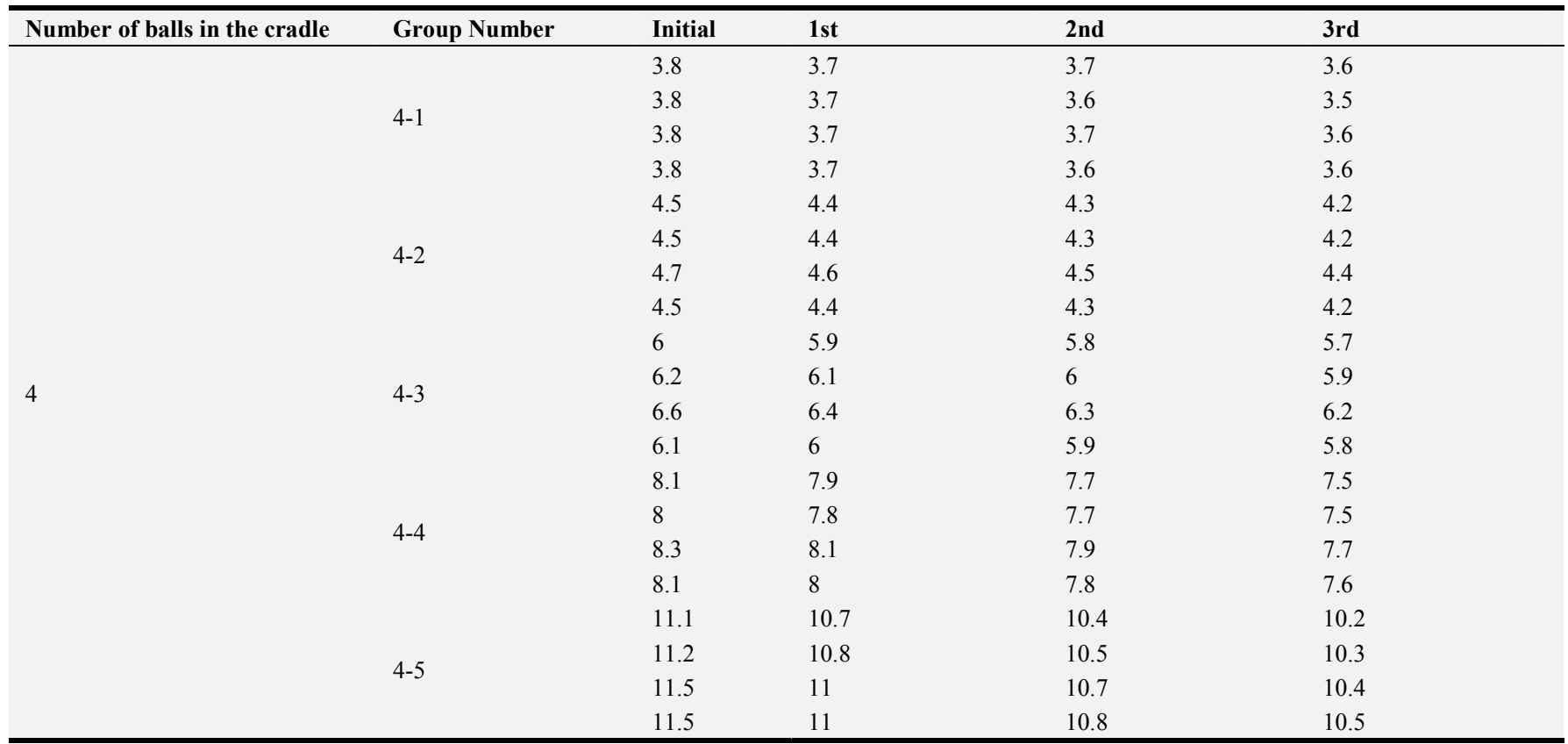

Table 9. The experiment data of Newton's Cradle of 5 balls.

\begin{tabular}{|c|c|c|c|c|c|}
\hline Number of balls in the cradle & Group Number & Initial & 1st & 2nd & 3rd \\
\hline \multirow{17}{*}{5} & \multirow{4}{*}{$5-1$} & 4 & 3.8 & 3.7 & 3.6 \\
\hline & & 3.8 & 3.7 & 3.6 & 3.5 \\
\hline & & 3.8 & 3.6 & 3.5 & 3.5 \\
\hline & & 3.8 & 3.7 & 3.6 & 3.6 \\
\hline & \multirow{3}{*}{$5-2$} & 4.5 & 4.4 & 4.3 & 4.2 \\
\hline & & 4.5 & 4.4 & 4.3 & 4.2 \\
\hline & & 4.7 & 4.6 & 4.5 & 4.4 \\
\hline & \multirow{4}{*}{$5-3$} & 6.5 & 6.2 & 6 & 5.8 \\
\hline & & 6 & 5.8 & 5.6 & 5.5 \\
\hline & & 6 & 5.9 & 5.7 & 5.5 \\
\hline & & 6 & 5.8 & 5.6 & 5.4 \\
\hline & \multirow{3}{*}{$5-4$} & 8 & 7.7 & 7.4 & 7.2 \\
\hline & & 8 & 7.7 & 7.4 & 7.2 \\
\hline & & 8 & 7.8 & 7.5 & 7.3 \\
\hline & \multirow{3}{*}{$5-5$} & 11.5 & 10.9 & 10.6 & 10.3 \\
\hline & & 11.5 & 11 & 10.7 & 10.4 \\
\hline & & 11.3 & 10.8 & 10.5 & 10.2 \\
\hline
\end{tabular}




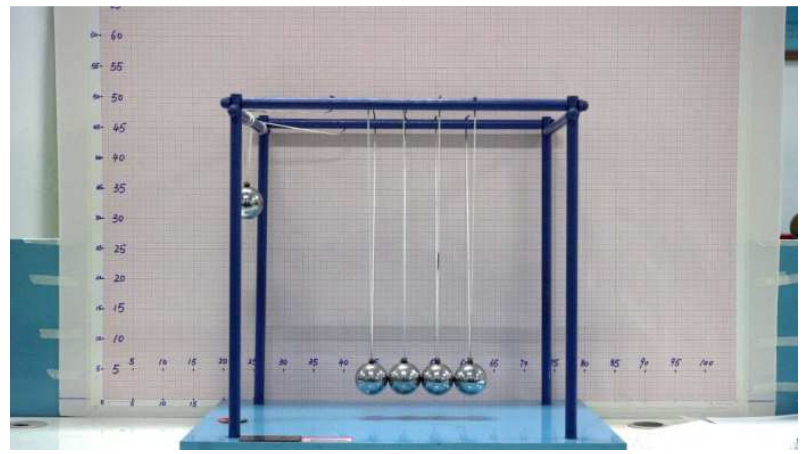

Figure 13. The 4 balls experiment of Newton's Cradle.

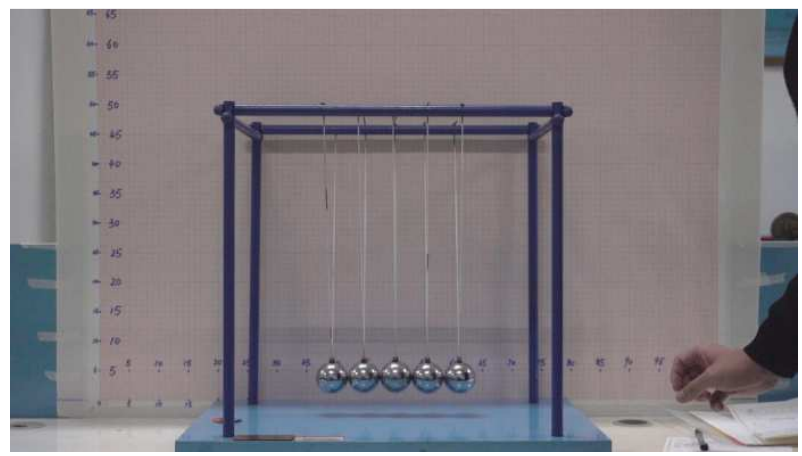

Figure 14. The 5 balls experiment of Newton's Cradle.

\section{References}

[1] Pengfei, L and Boris, M. (2014) Multisoliton Newton's cradles and supersolitons in regular and parity-time-symmetric nonlinear couplers. PHYSICAL REVIEW. [J] 2014 (89). p. 062926-1-062926-7. Available from: http://dx.doi.org/10.1103/PhysRevE.89.062926

[2] Paul, G. (2011) Two-ball Newton's cradle. PHYSICAL REVIEW. [J] 2011 (84). p. 067201-1-067201-4. Available from: http://dx.doi.org/10.1103/PhysRevE.84.067201

[3] Anissofira, A, et al. (2017) Newton's Cradle Experiment Using Video Tracking Analysis with Multiple Representation
Approach. Journal of Physics: Conference Series. [J] 2017 (895). p. 012107-1-012107-5. Available from: https://doi.org/10.1088/1742-6596/895/1/012107

[4] Zehao, W. (2019) The research about the momentum equation and the loss of energy in collision. Modern Business Trade Industry [J] 2019 (10). p. 192-194. Available from: http://www.cnki.net

[5] Qian, R and Jincai, D. (2003) STUDY ON "NEWTON' S CRADLE" AND ITS APPLICATION. JOURNAL OF VIBRATION AND SHOCK. [J] 22 (3). p. 78-79, 97. Available from: http://www.cnki.net

[6] Chenghui, W, et al. (2017) Transmission of Momentum and Energy in the System of Newton's Cradle. Physics Bulletin. [J] 2018 (6). P. 10-13. Available from: http://www.cnki.net

[7] Zhimin, P, et al. (2020) The research about the motion attenuation for Newton's Cadle. PHYSICS TEACHER. [J] 41 (4). p. 56-62. Available from: http://www.cnki.net

[8] Herrmann, F and Schmalzle, P. (1981) Simple Explanation of a Well-known Collision Experiment. American Journal of Physics. [J] 49 (8). p. 761-764.

[9] Herrmann, F and Seitz, M. (1982) How does the ball-chain work. American Journal of Physics. [J] 50 (11). p. 977-981.

[10] Cross, R, et al. (2017) Multiple collisions of two steel balls in Newton's cradle. European Journal of Physics. [J] 2017. p. 1-16. Available from: https://doi.org/10.1088/1361-6404/aa916, http://dx.doi.org/10.1103/PhysRevE.84.067201

[11] Judith, E and David, G. (1997) Newton's cradle and scientific explanation. The Physics Teacher. [J] 1997 (35). p. 411-417. Available from: http://dx.doi.org/10.1119/1.2344742

[12] David R, Alan V and Eugenia E 2009 Phys. Rev. Spec. Top.-Phys. Educ. Res. 5 1-13.

[13] Alan V 2001 Am J Phys [Internet]. vol; 69 (No. 11): 1139-46. Available from: http://dx.doi.org/10.1119/1.1399043

[14] LooK, Charles C, Giam H, Samuel T and Tat L 2012, Phys. Educ., vol. 47, no. 4, pp. 448-455.

[15] Jyoti B, Vandana S, Arbind J and O. S. K. S Sastri, 2015 Conference Paper. 